\title{
A CATALYTIC DECOMPOSITION OF CERTAIN PHENOL SILVER SALTS.
}

[FIRST PAPER. ${ }^{1}$ ]

\author{
By W. H. Hunter, A. O. Olson and E. A. Daniels. \\ Received July 8, 1916.
}

During the progress of work on the chromoisomeric silver salts of some halogenated phenols, it was observed by Torrey and Hunter ${ }^{2}$ that both the red and white silver salts of tribromophenol, if absolutely dry, reacted in a very unusual way with dry undiluted ethyl iodide. The iodide solutions rapidly assumed a deep blue color, which faded a little more slowly, giving way to a brownish yellow. A precipitate remained which consisted mainly of silver, bromide with a little iodide, while the ethyl iodide held in solution a white amorphous compound, which could be precipitated by alcohol. This compound could also be obtained by the use of other alkyl iodides, but the different preparations were practically identical in bromine content, showing that no alkyl group had been introduced. Direct tests showed a complete absence of silver and iodine.

At this point the work was dropped, as it offered no immediate assistance in the study of the silver salts. The reaction possessed considerable intrinsic interest however, because it was so different in all respects from the expected alkylation. On this account, work on the change has since been taken up in this laboratory, and the present paper embodies that part of the work which is concerned with the composition of the white amorphous compound, the preparation of analogous compounds, their genetic relation to the parent silver salts, and the nature of the reaction by which they are formed.

The Composition of the White Amorphous Compounds.-On taking up the work again, the amorphous compound from tribromophenol was analyzed for carbon and hydrogen, and its molecular weight was determined cryoscopically, when it was found that it possesses the formula $\left(\mathrm{C}_{6} \mathrm{H}_{2} \mathrm{Br}_{2} \mathrm{O}\right)_{n}$. Its physical properties are entirely in accord with this formula, as it is completely amorphous, and very soluble in organic liquids containing halogen, but swells up before dissolving, and on evaporation of the solvent is left as a yellow-brown varnish. Slowly heated in a melting point tube, it commences to shrink at about $260^{\circ}$, gradually sintering and turning darker, until at about $290^{\circ}$ it is almost black. On account of these properties it could only be purified by alternate solution in ethyl iodide or chloroform, and reprecipitation with alcohol, and its purity could only be tested by analysis of successive fractions obtained

${ }^{1}$ Some of the work described in this paper was presented in abstract at the Eighth International Congress of Applied Chemistry, New York, September, r912'.

2 This Journal, 33, 204 (Igii). 
in this manner. Until further information as to its constitution can be brought forward, it is suggested that it be called polydibromophenyleneoxide. ${ }^{1}$

Having ascertained the composition of the white amorphous compound, it became necessary to find out whether other compounds of analogous properties could be obtained from other phenols in like manner. For this purpose, as most like the tribromophenol, were chosen the symmetrical trichlorophenol, and the tribromoresorcinol monomethyl ether, which differs from tribromophenol only by a methoxyl group.

The silver salt of the last named phenol was found to behave in exactly the same way with alkyl iodides as that from tribromophenol, except that the blue color was more persistent. The oxide from this phenol proved to have a bromine content and molecular weight corresponding to that expected, and may be called polydibromomethoxyphenylene oxide. This silver salt soon proved to be very much better suited to a study of the decomposition than the tribromophenolate. For instance, simple covering of the salt with pure benzene is sufficient to induce the whole series of changes, starting in with the blue color, and ending with the formation of a solution of the amorphous oxide, which could be isolated by precipitation with gasoline.

On the other hand, silver trichlorophenolate gave but a slight abnormal reaction with cold ethyl iodide, forming almost no amorphous compound, but yielding trichlorophenetol instead. However, a short heating in benzene brought about the smooth decomposition of the salt, and there was obtained an oxide in every respect analogous to the others.

The Equation for the Reaction.-In these three cases, then, a compound had been obtained of a composition corresponding to the original silver salt minus a molecule of silver halide, but of very high molecular weight. In order to see if there might be any other products formed, a weighed sample of the silver salt from the resorcinol derivative was allowed to stand with cold ethyl iodide, and the reaction product was investigated. It was found that within the limit of error of the experi-

${ }_{1}$ This compound is probably identical with that obtained by Kastle and Loevenhart $(\mathrm{Am}$. Chem. J., 27, 34 (1902)) by the action of sunlight on a solution of tribromophenolbromide. To this compound, though colorless, Kastle and Gilbert (Am. Chem. J.,

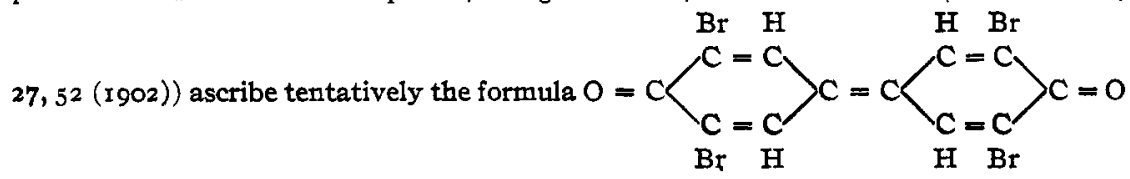
which is analogous to the formula now ascribed to the red compound known variously as "Lautemann's red," "diiodo phenylene oxide," and "tetraiodo diphenylene quinone." (Cf. Wiltsie, J. Soc. Chem. Ind., 19I0.) These interesting relationships will be discussed in a future paper. 
ment, the silver salt had been quantitatively transformed into amorphous oxide and tribromoresorcinol methyl ethyl ether, no other products being found.

Obviously, then, the ethyl iodide does not appear in the equation for the formation of the oxide, which will be as follows for the case of the silver tribromophenolate:

$$
n \mathrm{C}_{6} \mathrm{H}_{2} \mathrm{Br}_{3} \mathrm{OAg}=\left(\mathrm{C}_{6} \mathrm{H}_{2} \mathrm{Br}_{2} \mathrm{O}\right)_{n}+n \mathrm{AgBr}
$$

The Mechanism of the Decomposition.-An attempt at accounting for this action led to the formation of the following hypotheses:

I. The reaction may be intermolecular, the silver atom of one molecule uniting with a bromine from another molecule of the salt, to split out silver bromide and form a chain of rings.

2. The intermolecular reaction may be confined to two molecules, or a very few, which give a residue of low molecular weight, which polymerizes.

3. There may be an "intermediate addition" of alkyl halide, with the formation of a blue compound, and a subsequent splitting out of silver halide and alkyl halide. 'This would mean the formation of a simple molecule which could polymerize to the white oxide.

4. The decomposition may be strictly intramolecular, a decomposition of the silver salt itself, the silver atom uniting with a bromine atom from its own molecule, to leave the simple residue, which then polymerizes to form the oxide.

The hypotheses concerned with intermolectlar reactions seem to be eliminated by the following considerations:

I. The silver salt of the resorcinol derivative decomposes on wetting with benzene, which has very little solvent action on silver salts.

2. Ethyl iodide in vapor form acts on dry salt as if diluted with a solvent such as ether, bringing about an alkylation, with no decomposition. Evidently liquid ethyl iodide is necessary for the new change. It is equally unlikely that the salt dissolves in ethyl iodide.

3. There is no reason for supposing that silver could easily remove halogen attached to the ring, even if the salt were dissolved. Slight solution is more probable in alcohol, where ethyl iodide gives a normal alkylation.

4. There is no silver in the amorphous oxide, or in the blue resins left on evaporation of benzene solutions of the fresh reaction product. It is hard to see how every atom of silver could be removed from the amorphous compound by an intermolecular reaction, which would be expected to leave a silver atom on one end, and a bromine atom on the other end of a long chain of rings.

The next hypothesis, of an addition compound, was easily disposed of by showing that the smooth decomposition could be brought about by heat alone, or by warming to a slightly lower temperature, and "setting 
off" the salt with a minute amount of a reactive substance such as ethyl iodide or chlorine. The reaction would then proceed by its own heat, which proved to be sufficient to raise the temperature of one sample from $100^{\circ}$ to $180^{\circ}$.

Samples of the oxide produced at these high temperatures were analyzed for bromine. They proved to have a slightly lower content than the theoretical, giving a little over I \% less than the required amount of bromine. It is more surprising that the result should be so close than that a difference should exist, because it must be supposed that more deepseated changes would certainly occur at the temperatures reached in the decomposing mass of salt, and it would probably be especially difficult to remove such products entirely from the oxides. Nevertheless, there can be no doubt that even at this relatively high temperature, the formation of silver bromide and amorphous oxide is practically the only reaction occurring. A quantitative determination of reaction products proved impossible in this case, on account of the extreme fineness of the silver bromide formed, which became suspended in colloidal form in all solvents, and rendered the purification of the oxide very difficult. No other products than bromide and oxide, however, could be demonstrated in the reaction mixture, and only the low halogen content indicated the presence of other reactions.

These results show conclusively that the reaction can be brought about without any solvent, by heat alone. They also show that reactive substances have a fuse-like action on salt which is heated to a temperature just insufficient to bring about decomposition of the type studied.

This fuse-like action is probably entirely analogous to the decomposition at low temperatures, which is seen in its extreme form in the decomposition of the silver salt of the monomethyl ether of tribromoresorcin on covering with thiophene-free benzene in a desiccator. Light is apparently not necessary for this change, as it starts first in any deep crack in a lump of salt, which on being opened, will be found completely filled with blue solution. It seems to be due to contact of the benzene with the salt, so that we have the unusual case of a liquid catalyzing a change occurring in a homogeneous solid. Since the deep color is reminiscent of Vorländer's addition compounds of type $A$, it may be well to insist again upon the absence of silver in the blue varnishes obtained, and upon the fact that the color of these varnishes goes over completely into the light yellow of the amorphous resin left by solutions of the oxides. Further, the action of ethyl iodide vapor in giving the ether instead of the amorphous oxide, while benzene causes the oxide formation, seems to indicate that the presence of actual liquid is more important than the chemical individual it represents. Up to the present time, no experiments have been devised, which can settle the question as to whether the solid dissolves somewhat, 
the reaction then occurring in solution, or whether strong adsorption of the benzene or ethyl iodide occurs, followed by decomposition of the surface layers of the solid. This is one of the most interesting points about the change, and it is hoped that some way may be found by which the precise action of benzene may be established. It would be especially interesting in view of Langmuir's late work on adsorption and heterogeneous reactions, on account of the fact that the crystalline white silver salt of tribromophenol reacts just as does the apparently amorphous red form. It does not seem wise to take up this phase of the problem until all other parts of the work shall have been cleared up as far as possible.

The reaction is somewhat like those smooth decompositions of silver salts studied by Angeli and others, ${ }^{1}$ one of which they represent by the equation $\mathrm{C}_{6} \mathrm{H}_{5} \mathrm{~N}\left\langle_{\mathrm{N}=\mathrm{O}}^{\mathrm{OAg}}=\mathrm{Ag}+\mathrm{NO}+\mathrm{C}_{6} \mathrm{H}_{5} \mathrm{NO}\right.$. It must be sharply contrasted with that of Burke and Donnan, ${ }^{2}$ who found that alkyl halides in alcohol act on silver nitrate to give two reactions, represented by the equations

$$
\begin{gathered}
\mathrm{C}_{2} \mathrm{H}_{5} \mathrm{I}+\mathrm{AgNO}_{3}+\mathrm{C}_{2} \mathrm{H}_{5} \mathrm{OH}=\mathrm{AgI}+\left(\mathrm{C}_{2} \mathrm{H}_{5}\right)_{2} \mathrm{O}+\mathrm{HNO}_{3} \\
\mathrm{C}_{2} \mathrm{H}_{5} \mathrm{I}+\mathrm{AgNO}=\mathrm{AgI}+\mathrm{C}_{2} \mathrm{H}_{5} \mathrm{NO}_{3} .
\end{gathered}
$$

Several interesting problems are raised by the acceptance of the simple decomposition, some of which are still being investigated. It seems worth while to mention two of them at this point.

The first one is that of the blue color. So far, no expedient has been devised, which permits the isolation of a blue compound. All the data so far at hand lead to the view that the blue compound is a polymer of the unsaturated residue, lower than the white oxide-even conceivably the unsaturated residue itself-or that it may be related to coerulignone.

Far more important is the following problem: If the hypothesis of a simple monomolecular reaction is correct, how can it be brought about in one case by heat, in another case by a reactive substance such as ethyl iodide, that can and does react with other molecules of the phenolate at the same time, and in yet a third case by a substance, benzene, which presumably does not react with it in the ordinary sense of the word?

It may, perhaps, be temporarily viewed as a result of at least two factors, of which one is the known instability of silver salts, and the other the presence in the molecule of a benzene ring loaded with halogen atoms in the critical ortho and para positions. It may be conceived that in such a molecule, the silver atom would be unusually closely related to one of the halogens, whether by being actually on the same carbon atom, as sug-

${ }^{1}$ Angeli and Alessandri, Atti accad. Lincei, 18, 38 (1909); 18, 784 (1910); Angeli and Marchetti, Ibid., I7, 695 (1908).

${ }^{2}$ Trans. Chem. Soc., 85, 555 (1904); Zeit. physik. Chem.; 69, 148 (1909). 
gested for the colored forms by Torrey and Hunter, ${ }^{1}$ or better in some other way, which could be equally applicable to all of these salts. In such a molecule there would be considerable tendency for decomposition to occur in such a way as to split out silver bromide in just such a manner as occurs with our salts. In other words, there would be a strain in the molecule, which would not be sufficient to bring about a spontaneous decomposition of the pure dry salt, but which would determine the line of fission of the molecule when gently heated.

When the salt is not heated, but is covered with benzene, the effect of solution, or, as has been already suggested, of adsorption -in either case, the intimate approach of molecules of liquid and salt, must bring about changes comparable to those of ordinary surface catalytic effects-perhaps comparable more nearly to Baly's ideas of chemical change.

Further work is in progress along several lines opened up by this view of the salts and their decomposition, which is intended to serve at present simply as a working hypothesis.

\section{Summary.}

The silver salts of the following phenols<smiles>OC1CC(Br)CC1Br</smiles><smiles>CO[C@@H]1C[C@H](Br)C[C@@H]1Br</smiles><smiles>OC1CC(Cl)CC1Cl</smiles>

suffer a smooth decomposition into silver halide and an amorphous polymerized residue, in the sense of the following equation:

$$
n \mathrm{C}_{6} \mathrm{H}_{2} \mathrm{Br}_{3} \mathrm{OAg}=n \mathrm{AgBr}+\left(\mathrm{C}_{6} \mathrm{H}_{2} \mathrm{Br}_{2} \mathrm{O}\right)_{n}
$$

This occurs readily on suspension of the salt in alkyl iodides, or in pure benzene.

The same effect is produced by heating to temperatures slightly above $100^{\circ}$.

It is suggested by the authors that this may be due to a stress existing in the molecule of the silver salt, between the silver atom and the halogen which goes out with it, not sufficient to cause decomposition until some catalytic effect is exerted on the molecule from without.

\section{Experimental Part.}

Preparation of the Silver Salts of the Phenols.-Since the phenols used are but slightly soluble in water, but give soluble alkali salts, quite strongly hydrolyzed, the following procedure has been used for the preparation of their silver salts:

The pure phenol was dissolved in a slight excess of twice normal potassium hydroxide solution, as free from halogen as possible, and after considerable dilution $\mathrm{r} \%$ acetic acid was added to the mixture with

${ }^{3}$ Loc. cit. 
stirring, until a slight permanent precipitate of the phenol was obtained, indicating a neutralization of the excess alkali. A few drops of very dilute silver nitrate were then added, and the solution was well stirred to insure opportunity for the precipitation of any halogen ion present.

The mixture was then allowed to stand for a while, and filtered without suction. A clear bright solution resulted, which gave a voluminous precipitate of the desired silver salt on addition of silver nitrate. The silver salts of the three phenols used in this work were all known-the one from trichlorophenol is yellow, the one from tribromoresorcinol monomethyl ether is white, and the one from tribromophenol is one of Torrey's ${ }^{1}$ silver salts, and exists in two modifications, a red and a white.

This precipitate was allowed to stand in the dark for an hour or more, to allow it to become granular, and then filtered without suction, with washing on the filter till the wash water gives only a slight opalescence on testing for silver--the silver salts themselves are slightly soluble in water. It was then transferred to a Buechner funnel, sucked dry, partly dried by continuing the suction, and transferred to clean glass watch glasses, spread out thin. It was allowed to dry by standing in clean air in the dark, and finally in a desiccator in the dark, best over calcium chloride. In this way very pure samples of silver salts were obtained, which could be kept for a long time without signs of change.

Experiments with Tribromophenol Derivatives.-Some of the polydibromophenylene oxide was made by the action of ethyl iodide on the red silver salt of tribromophenol, as described by Torrey and Hunter. ${ }^{2}$

Subst. o.2187; $\mathrm{CO}_{2}, 0.2348 ; \mathrm{H}_{2} \mathrm{O}$, o.0199. Subst. o.I500; $\mathrm{AgBr}$, o.226o.

Calc. for $\mathrm{C}_{6} \mathrm{H}_{2} \mathrm{Br}_{2} \mathrm{O}: \mathrm{C}, 28.8 \mathrm{I} ; \mathrm{H}, \mathrm{I} .02 ; \mathrm{Br}, 63.99$. Found: C, 29.28; H, I.02; $\mathrm{Br}, 64.12$.

The silver halide from the Carius determination was treated with zinc and sulfuric acid, and the solution tested for iodide with chloroform and bromine water, but none was found.

Molecular-weight Determination. This was carried out with recrystallized bromoform as solvent. $\mathrm{K}=144$. (Freezing point.)

Solvent, 30.19 g.; subst. $0.207,0.332 ; \Delta, 0.008^{\circ}$, 0.024; Mol. wt., I 2,400, 6,600.

Decomposition of Silver Trichlorophenolate. ${ }^{3}$-About two grams of the silver salt were placed in an Erlenmeyer flask with $50 \mathrm{cc}$. of benzene, and the mixture was heated under a reflux condenser on a water bath at $60^{\circ}$ for about thirty minutes. The solid remaining at the end of this time was light gray in color, and very finely divided. A great deal of trouble was encountered in obtaining a clear solution, but this was finally accomplished by many filtrations without suction. The clear benzene solution

1 Torrey and Hunter, This Journal, 33, I94 (r9II); Bèr., 40, 4322 (IgO7); Hantzsch, Ibid., 40, 4875 (Ig07); Kruyt, Ibid., 43, 543 (I9ro).

2 This Journal, 33, 204 (I9II).

3 The work on trichlorophenol was performed by Mr. L. Karatz. 
was then completely precipitated by gasoline. An amorphous compound entirely similar in appearance to the one from tribromophenol was obtained, which was purified by alternate solution and reprecipitation with benzene and gasoline and analyzed for chlorine.

Subst. $0.1236,0.1030,0.2186$ and $0.2008 ; \mathrm{AgCl}, 0.2221,0.1804,0.3896$ and 0.3565 .

Calc. for $\mathrm{C}_{6} \mathrm{H}_{2} \mathrm{Cl}_{2} \mathrm{O}: \mathrm{Cl}, 44.09$. Found: $44.74,43.6 \mathrm{I}, 44 . \mathrm{II}, 43.98$. Av. $=44 . \mathrm{Ir}$.

Action of Ethyl Iodide. - The chief effect of ethyl iodide is the formation of the trichlorophenetol. Very little amorphous oxide can be obtained in this way, but it can be demonstrated as a reaction product.

Work on the Silver Salt of Tribromoresorcinol Monomethylether.

Decomposition by Ethyl Iodide.-On covering the dry salt with ethyl iodide the characteristic blue color developed very much more quickly than with the tribromophenol salt, and lasted very much longer. The amorphous white compound was easily obtained by precipitating it with alcohol, though it was at first colored green by adhering blue compound.

Subst. 0.1486; $\mathrm{AgBr}$, 0.1998. Calc. for $\mathrm{C}_{6} \mathrm{H}\left(\mathrm{OCH}_{3}\right) \mathrm{Br}_{2} \mathrm{O}: 57.1 \mathrm{r}$. Found: 57.25 .

In order to see if any other products were formed, than silver bromide, iodide, the oxide, and the methyl ethyl ether of tribromoresorcinol, $0.8 \mathrm{I}$ I g. of the salt was covered with pure ethyl iodide, and allowed to stand twenty-four hours. The mixture was then separated by filtration, and the silver salts were washed well, the filtrate and washings being received in a weighed beaker.

The residual silver salt weighed $0.36 \mathrm{I} \mathrm{g}$.

The mixture of oxide and ether was heated in the flask in boiling water, but could not be brought to a constant weight, apparently because of slow volatilization of the ether, which crystallized out on the walls of the flask. The observed weight of these products was $0.546 \mathrm{~g}$. No products other than ether and oxide were found.

Now, if all the salt had been converted into oxide and silver bromide, the latter would have weighed $0.326 \mathrm{~g}$., and the oxide, $0.485 \mathrm{~g}$.

This excess in weight of silver residue is due to the weight of iodine in excess of the equivalent bromine, $\mathrm{I}-\mathrm{Br}=0.35 \mathrm{~g}$.

The total excess weight of both residues is equal to the whole of the ethyl iodide added to the salt, and is $0.096 \mathrm{~g}$.

Calculated from the silver residue, this should be $0.116 \mathrm{~g}$., if only these two reactions occurred.

This is two centigrams of error in ninety-one centigrams, not twenty per cent. of error, and since a loss of the phenol ether from the organic residue would account for part of this, by diminishing the total excess weight, it is not surprising that the result is not closer. Also, some silver bromide might have been transformed into iodide by standing twentyfour hours in ethyl iodide, and this would have increased the silver residue, 
but would also have increased over much the calculated weight of ethyl iodide added. Further, no other products have ever been isolated from these reaction mixtures.

Tribromoresorcinol Methyl Ethyl Ether.-This is formed at the same time as the amorphous oxide, and can be obtained from the filtrate after precipitation of the oxide by alcohol. Long, hard needles from alcohol and water. Melts at $75^{\circ}$. Soluble in ordinary solvents.

Analysis for bromine:

Subst. 0.2593 and $0.325 \mathrm{I} ; \mathrm{AgBr}, 0.3756$ and 0.4700 .

Cale. for $\mathrm{C}_{6} \mathrm{HBr}_{3}\left(\mathrm{OCH}_{3}\right)\left(\mathrm{OC}_{2} \mathrm{H}_{5}\right)$ : 61.67. Found: 61.64, 6r.5o.

Molecular-weight Determination on the White Oxide.

This was carried out, using benzene as solvent, by the cryoscopic method. Solvent, I6.0 g. Subst. $0.3530,0.2573 ; \Delta 0.049^{\circ}, 0.011^{\circ}$. Mol. wt. $2250,7308$.

\section{Decompositions at Low Temperatures.}

Silver Salt of Tribromoresorcinol Monomethyl Ether: Action of Benzene.-A pure, dry sample of silver salt was covered with thiophene-free benzene, and placed in a calcium chloride desiccator. In a short while a blue color developed, starting first at the larger lumps, and then diffusing away into the solution, which finally became very deep blue. No particular catalytic effect seemed to be exerted by the blue in solution. It was filtered from the solid residue, and the filtered solution was allowed to evaporate at ordinary temperature. The blue color slowly disappeared, and when the benzene had evaporated, a reddish yellow varnish was left, which had all the properties of the amorphous oxides.

A most interesting observation was made on a very pure sample of silver salt, which was washed as usual, and then heated with benzene before it had become entirely dry. The blue color appeared in this case also, and rather rapidly. Another pure sample was then heated with benzene. The blue color appeared immediately, and the same cycle of changes occurred, in much more rapid sequence. The filtered benzene solution, after disappearance of the blue color, gave a copious white precipitate of amorphous substance on the addition of either alcohol or ligroin.

Cold Ethyl Iodide Vapor.-0.1927 g. of dry salt was placed in a watch glass, and this suspended in a beaker a couple of inches above the bottom, which was covered with ethyl iodide. It was allowed to stand about seventeen hours, removed to the balance case, and weighed in one hour. It had gained $0.047 \mathrm{I} \mathrm{g}$. in this time. Calculated gain for complete ether formation, $0.0642 \mathrm{~g}$.

On investigation of the product, it was found that no recognizable amount of amorphous compound was present, but that the methyl ethyl ether of tribromoresorcinol was present.

Ethyl Bromide.-Some very pure ethyl bromide was made free from alcohol and ether and used soon after its manufacture. 
It gave no blue color with Torrey's silver salts from tribromophenol on standing.

A sample of the silver salt of tribromoresorcinol monomethyl ether was covered with the ethyl bromide, and placed in a desiccator. After a time, the blue color developed where the salt was piled up highest.

Another sample partly in lumps was placed in the ethyl bromide without crushing. The blue color appeared first in the lumps.

It is interesting that ethyl bromide appears to stand nearer to benzene in its catalytic action than to ethyl iodide.

\section{Decompositions at Higher Temperatures.}

A thin layer of the silver salt was placed in a narrow porcelain boat about five inches long, and this was placed in the inner tube of a condenser. Steam was passed through the outer jacket of the condenser, and a thermometer was introduced near the salt. It soon registered over $90^{\circ}$, and in seven minutes had reached $99.5^{\circ}$. It was removed, and the salt heated about a minute longer. Then a flask containing pure ethyl iodide was attached to the condenser, and warmed. Immediately, even before the ethyl iodide had started to boil, a greenish blue patch appeared on the salt nearest the ethyl iodide. In a second or two, the blue color changed to a dull brown, and then this color spread rapidly away from this end of the boat, in one experiment requiring about five seconds to go twenty-five millimeters. An entirely solid residue was left in the boat, though the reaction was accompanied by a curious appearance of fusing or sintering of the salt. Fine particles were also seen to fly up a little distance from the mass, so several samples were weighed out, to see if any amount of substance was being lost in this way, or as a gas.

$0.7455 \mathrm{~g}$. lost $0.0047 \mathrm{~g}$. $0.8390 \mathrm{~g}$. lost $0.003^{2} \mathrm{~g}$. $0.6540 \mathrm{~g}$. lost $0.005 \mathrm{r}$ g.

Another weighed sample was heated alone, without treatment with ethyl iodide. It did not decompose.

$0.4426 \mathrm{~g}$. gained $0.0018 \mathrm{~g}$.

It was then treated with iodide vapor as the other samples, when it decomposed, and gained $0.0013 \mathrm{~g}$. more.

The same experiments were tried, using chloroform vapor instead of steam, and the same decomposition was found to occur.

Another sample was lightly packed into a pear-shaped bulb made from a test tube, and a thermometer bulb was inserted into the middle of the salt. It was then heated with live steam until the thermometer registered $99.5^{\circ}$, when a very small amount of ethyl iodide was squirted into the bulb so as to touch the silver salt. This decomposed immediately as before, and very soon the temperature commenced to rise, until it reached a maximum of $185^{\circ}$.

Experiments in Tubes.-Pieces of ordinary glass tubing, $125^{-1} 5^{\circ} \mathrm{mm}$. long, were sealed at one end and cleaned thoroughly. One was filled 
with silver salt, and then placed in the steam jacket. After five minutes of heating, the ethyl iodide was let in. The expected green patch appeared at the open end, and then the decomposition proceeded through the tube as in the boat.

Another tube was half filled with silver salt, a layer of sand was placed on it, four or five millimeters in thickness, and the rest of the tube was then filled with more silver salt. The reaction proceeded to the sand only.

In another tube, even less sand was used, the layer being but two or three millimeters thick, in order to avoid contact between the two divisions of the salt. The action again ceased at the sand.

Several samples of these residues were combined and extracted with benzene. This solution was treated with fullers' earth, filtered, and ligroin was added to the reddish solution, when a pink precipitate was obtained. This was redissolved in benzene and reprecipitated six times, and the final product, still slightly pink, was analyzed for halogen.

Subst. 0.1655 and $0.1595 ; \mathrm{AgBr} 0.2179$ and 0.2100.

Calc. for $\mathrm{C}_{6} \mathrm{H}\left(\mathrm{OCH}_{3}\right) \mathrm{Br}_{2} \mathrm{O}: \mathrm{Br}$. 57.I I. Found: $56.03,56.03$.

\section{Effect of Other Substances.}

The following substances, in small amounts, were found to cause the decomposition of preheated salt: Propyl bromide, chloroformic ester, concentrated hydrochloric acid, fuming nitric acid, iodine, and bromine.

Minneapolis, Minnesota.

\section{HYDRAZINO DIACIDS.}

\section{BY J. R. BAILEY AND L. A. Mreesea}

Received June 12, 1916.

\section{Introduction.}

The first hydrazino diacid, hydrazinodiacetic acid, $\mathrm{NH}_{2} \mathrm{~N}\left(\mathrm{CH}_{2}-\right.$ $\mathrm{COOH})_{2}$, was discovered in the Heidelberg laboratory by Curtius and Hussong. ${ }^{1}$ The method of preparing this substance was improved by Bailey and Read and its reactions further investigated. ${ }^{2}$ Darapsky and Prabhakar have announced the discovery of hydrazinodimalonic acid, ${ }^{3}$ the second hydrazino diacid thus far reported, but the work described in this article shows that the proofs deduced for the constitution of this substance are not valid.

Hydrazinodiacetic acid is readily made by the action of monochloroacetic acid on hydrazine in alkaline solution, and as the product is difficultly soluble in water, its isolation presents no difficulty. It might be expected that other halogen acids would produce similar results with hydrazine, and that the solubilities in water of the hydrazino diacids thus formed

1 J. prakt. Chem., [2] 83, 249 (1911).

2 This Journal, 36, I748 (I9I4).

${ }^{3}$ Moreshwar Prabhakar, Dissertation, Heidelberg, 1912. 